\title{
CD47 antibody helps phagocytes fight paediatric cancer
}

\section{C4 \\ Blocking the CD47-SIRPa signalling axis suppresses the 'don't eat me' signal}

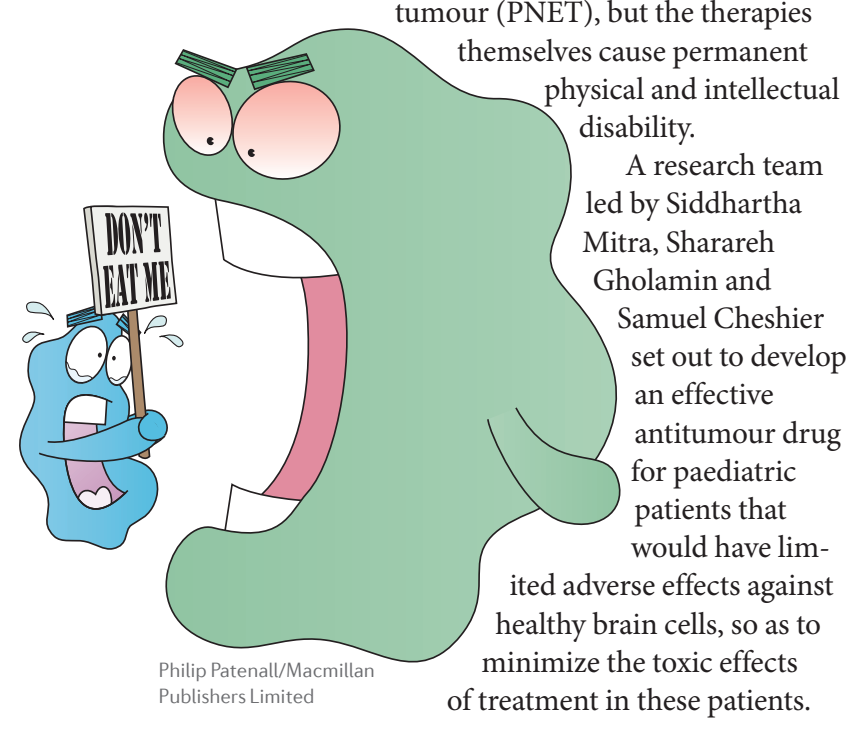

The team investigated the effect of blocking CD47, a protein that enables some cancer cells to evade the innate immune system by binding to signal regulatory protein $\alpha(\operatorname{SIRP} \alpha)$, an inhibitory receptor present on the surface of myeloid cells. "The binding of CD47 to SIRPa imparts a 'don't eat me' signal to macrophages," explains Mitra. "Blocking the CD47-SIRPa signalling axis suppresses the 'don't eat me' signal and enables efficient phagocytosis in all cancers tested to date. Normal healthy cells do not express the requisite 'eat me' signals; thus, blockade of CD47-SIRPa interaction does not induce phagocytosis of normal cells."

The researchers made a mouse xenograft model of paediatric brain cancer by transplanting medulloblastoma cells into the cerebellum of immunodeficient mice that lacked $\mathrm{B}$ cells, T cells and natural killer cells, but had phagocytic macrophages and microglia. Treatment of these mice with a humanized anti-CD47 antibody (Hu5F9-G4) resulted in reduced tumour volume, clearance of leptomeningeal metastatic disease and increased survival compared with mice that received a control treatment. Immunohistochemical analysis of the xenograft mouse brains showed recruitment of macrophages in Hu5F9-G4-treated mice, but not in control-treated mice. Furthermore, Hu5F9-G4 reduced tumour volume and increased survival in mice with grafts of ATRT, PNET, DIPG and paediatric glioblastoma tumour cells.
Importantly, the team showed that Hu5F9-G4 treatment did not result in phagocytosis of normal brain cells: in a mixed culture of medulloblastoma cells, normal human neuronal progenitor cells (NPCs) and macrophages, Hu5F9-G4 treatment resulted in a sevenfold higher phagocytosis rate of tumour cells than of NPCs.

To determine how other immune cells might affect the safety and efficacy of the treatment, the investigators examined the effect of blocking CD47 signalling in an immunocompetent system. In wild-type mice implanted with a mouse high-grade glioma cell line, treatment with a mouse anti-CD47 antibody was associated with reduced tumour size and increased survival. Immunostaining of the brains of these mice showed that anti-CD47 antibody treatment was associated with an increase in the number of macrophages around the tumour site, but no evidence of neuronal damage or gliosis was detected.

These findings indicate that anti-CD47 antibodies could provide a much-needed safe treatment for malignant paediatric brain tumours. The team are now looking ahead to human studies and plan to test the safety of anti-CD47 antibodies in phase I clinical trials.

Charlotte Ridler

ORIGINAL ARTICLE Gholamin, S. et al Disrupting the CD47-SIRP $\alpha$ anti-phagocytic axis by a humanized anti-CD47 antibody is an

efficacious treatment for malignant pediatric brain tumors. Sci. Transl. Med. 9, eaaf2968 (2017) 